\title{
Intrinsic ferroelectric polarization of orthorhombic manganites with $E$-type spin order
}

\author{
Y. S. Chai, ${ }^{1}$ Y. S. Oh, ${ }^{1}$ L. J. Wang, ${ }^{2}$ N. Manivannan, ${ }^{1}$ S. M. Feng, ${ }^{2}$ Y. S. Yang, ${ }^{1}$ L. Q. Yan,,${ }^{1,2}$ C. Q. Jin, ${ }^{2}$ and Kee Hoon Kim ${ }^{1, *}$ \\ ${ }^{1}$ CeNSCMR, Department of Physics and Astronomy, Seoul National University, Seoul 151-747, Republic of Korea \\ ${ }^{2}$ Institute of Physics, Chinese Academy of Sciences, P.O. Box 603, Beijing 100190, P. R. China \\ (Received 14 August 2011; revised manuscript received 18 September 2011; published 7 May 2012)
}

\begin{abstract}
By directly measuring electrical hysteresis loops using the Positive-Up Negative-Down (PUND) method, we determined accurately the remanent ferroelectric polarization $P_{r}$ of orthorhombic $R \mathrm{MnO}_{3}(R=\mathrm{Ho}, \mathrm{Tm}, \mathrm{Yb}$, and $\mathrm{Lu}$ ) compounds below their $E$-type spin ordering temperatures. We found that $\mathrm{LuMnO}_{3}$ has the largest $P_{r}$ of $0.17 \mu \mathrm{C} / \mathrm{cm}^{2}$ at $6 \mathrm{~K}$ in the series, the value of which allows us to predict that its single-crystal form can produce a $P_{r}$ of at least $0.6 \mu \mathrm{C} / \mathrm{cm}^{2}$ at $0 \mathrm{~K}$. Furthermore, at a fixed temperature, $P_{r}$ decreases systematically with increasing rare earth ion radius from $R=\mathrm{Lu}$ to Ho, exhibiting a strong correlation with the variation of the in-plane $\mathrm{Mn}-\mathrm{O}-\mathrm{Mn}$ bond angle and $\mathrm{Mn}-\mathrm{O}$ distances. Our experimental results suggest that the contribution of the $\mathrm{Mn} t_{2 g}$ orbitals may dominate the ferroelectric polarization.
\end{abstract}

DOI: 10.1103/PhysRevB.85.184406 PACS number(s): 75.85.+t, 77.80.-e, 75.50.Ee, 75.47.Lx

\section{INTRODUCTION}

Recent intensive researches on multiferroic materials are motivated by great interests in the fundamental physics of spin-lattice coupling as well as the potential for using these materials in the multifunctional memories and sensors. ${ }^{1-4}$ An interesting class of multiferroic materials that have been studied intensively in recent years is the so-called magnetic ferroelectrics. In these materials the ferroelectric polarization $(P)$, induced by the primary magnetic order, can be sensitively tuned by magnetic fields through the control of magnetic states. ${ }^{5}$ It is now well known that both collinear and noncollinear spin orderings in these magnetic ferroelectrics can generate a nontrivial $P$ through several mechanisms such as exchange striction ${ }^{6,7}$ and the inverse Dzyaloshinskii-Moriya interaction. ${ }^{8,9}$ Limited by the rather weak spin-lattice coupling strength set by these mechanisms, most of the magnetic ferroelectrics studied so far exhibit $P$ values less than $0.1 \mu \mathrm{C} / \mathrm{cm}^{2}$, which is much smaller than that of conventional ferroelectrics.

On the other hand, several theoretical works have suggested the possibility of achieving large $P$ in orthorhombic $(o)$ $R \mathrm{MnO}_{3}(R=\mathrm{Ho}, \mathrm{Er}, \mathrm{Tm}, \mathrm{Yb}$, and $\mathrm{Lu})$ with a collinear $E$ type antiferromagnetic (AFM) spin order. ${ }^{10-13}$ In this system $\mathrm{Mn}^{3+}$ has a $t_{2 g}^{3} e_{g}^{1}$ configuration and thus undergoes an $e_{g}$ orbital ordering with the Jahn-Teller distortion of the $\mathrm{MnO}_{6}$ octahedra, leading to a distribution of long and short $\mathrm{Mn}-\mathrm{O}$ bond lengths $\left(d_{l}\right.$ and $\left.d_{s}\right)$ in the $a b$ plane [Fig. 1(a)]. ${ }^{14}$ The $E$-type AFM order at low temperatures is predicted to induce $P$ by two different mechanisms. First, the ionic displacement polarization $P_{\text {ion }}$ is induced by the competition between the ferromagnetic super-exchange interaction between $e_{g}$ orbitals and the AFM interaction between $t_{2 g}$ orbitals through the inverse Goodenough-Kanamori rules, ${ }^{10,11}$ as illustrated in Fig. 1(b). Second, the electronic polarization $P_{\text {ele }}$ results from selective electron hopping between orbitals with parallel spins and has contributions from both $t_{2 g}$ and $e_{g}$ orbitals as well as oxygen, although the $t_{2 g}$ and $e_{g}$ orbital contributions nearly cancel each other, leaving just mainly the oxygen contribution in the end. As summarized in Fig. 1(b), ${ }^{10}$ the $t_{2 g}$ and $e_{g}$ contributions in both mechanisms have opposite signs, and the total $P$ results from the sum of those contributions. Both model
Hamiltonian ${ }^{12}$ and first-principles ${ }^{10}$ calculations predicted that a total $P$ up to approximately $6 \mu \mathrm{C} / \mathrm{cm}^{2}$ can be obtained in $o-\mathrm{HoMnO}_{3}$, and the value is almost the same over the $o-R \mathrm{MnO}_{3}$ series (from $R=\mathrm{Ho}$ to $\mathrm{Lu}$ ). In a subsequent study using the hybrid functional approach, the predicted value decreased to approximately $2 \mu \mathrm{C} / \mathrm{cm}^{2}$. This reduction occurred because the hybrid functional method reduces the electronic contribution. ${ }^{15}$

An experimental test of these theoretical results is an important and necessary step in multiferroic research as it can not only identify the maximum allowed $P$ when the $E$-type spin order is present but may also help find yet another new compound generating large $P$. However, previous experimental studies on the $o-R \mathrm{MnO}_{3}$ compounds have shown inconsistent results. Early studies on $o-\mathrm{HoMnO}_{3}$ and $o-\mathrm{TmMnO}_{3}$ revealed $P$ values inside the $E$-type AFM phase of approximately $0.008 \mu \mathrm{C} / \mathrm{cm}^{2}$ at $5 \mathrm{~K}$ and 0.15 $\mu \mathrm{C} / \mathrm{cm}^{2}$ at $2 \mathrm{~K}$, respectively. ${ }^{16,17}$ More recently, $P$ values of $0.07-0.09 \mu \mathrm{C} / \mathrm{cm}^{2}$ were observed at $2 \mathrm{~K}$ for all $o-R \mathrm{MnO}_{3}$ $\left(R=\mathrm{Ho}, \mathrm{Tm}, \mathrm{Yb}, \mathrm{Lu}\right.$ and $\left.\mathrm{Y}_{1-y} \mathrm{Lu}_{y}, y=0-1\right)$, which changed little with variation in the rare earth ion radius $\left(r_{R}\right) .{ }^{18} \mathrm{It}$ should be noted that all of these previous reports employed the pyroelectric current $\left(J_{p}\right)$ measurement. In this method, the temperature-dependent $J_{p}$ is measured upon warming after applying dc electric poling field from a high temperature above the ferroelectric Curie temperature to the low temperature at which the measurement starts. The temperature-dependent polarization $P_{\mathrm{dc}}(T)$ can be obtained by integrating $J_{p}$ as a function of time. However, the common procedures followed in this method can be susceptible to several experimental challenges. First, most of the $o-R \mathrm{MnO}_{3}(R=\mathrm{Ho}$ to $\mathrm{Lu})$ compounds have a polycrystalline pellet form synthesized under high pressure. Because of this, the $J_{p}$ measurement is subject to incomplete electric poling, and thus the ferroelectric domains can be randomly oriented. Second, the electric poling process usually produces space charges that can be trapped at the polycrystalline grain boundaries, providing a spurious $P$ contribution. These experimental difficulties in determining the $P$ value in polycrystalline ferroelectrics can be greatly reduced by employing the so-called Positive-Up Negative-Down (PUND) method, ${ }^{19}$ which has been extensively employed 
(a)

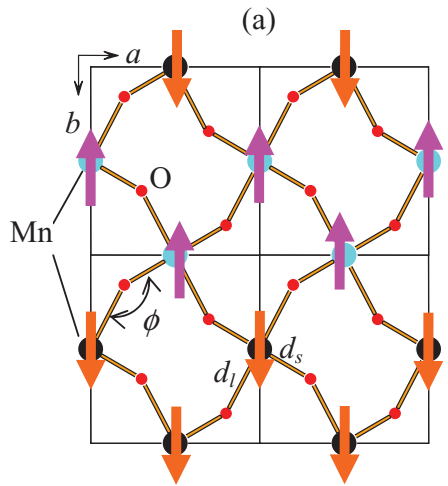

(b)

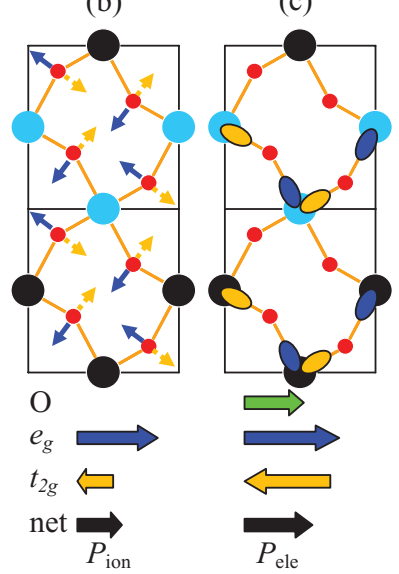

FIG. 1. (Color online) (a) $a b$ plane arrangement of $\mathrm{Mn}$ and $\mathrm{O}$ atoms and spins in the $E$-type spin order realized in $o-R \mathrm{MnO}_{3}$. (b) The arrows represent the $\mathrm{O}$ atom displacement due to $e_{g}$ (solid) and $t_{2 g}$ (dashed) orbitals. (c) The ellipses indicate the Mn charge deviations due to the $e_{g}$ (along $d_{l}$ ) and $t_{2 g}$ (along $d_{s}$ ) hoppings, as described in Ref. 10. Arrows at the bottom depict the theoretical predictions in Ref. 10 for the resultant polarizations from each contribution categorized into $P_{\text {ion }}$ and $P_{\text {ele }}$.

in ferroelectric thin film researches and recently has been applied to multiferroic single crystals. ${ }^{20}$ In particular, we have recently used this technique to prove that a polycrystalline $o-\mathrm{HoMnO}_{3}$ sample has an intrinsic $P$ of approximately $0.24 \mu \mathrm{C} / \mathrm{cm}^{2},{ }^{21}$ which is much smaller than that predicted by band calculations. ${ }^{10,12,15}$

In this paper we report our systematic efforts to determine accurately the $r_{R}$-dependent ferroelectric polarization of $o-R \mathrm{MnO}_{3}$ for $R=\mathrm{Ho}, \mathrm{Tm}, \mathrm{Yb}$, and Lu with $E$-type spin order. By applying the PUND method to high quality specimens that have been well characterized by structural studies, we succeeded in measuring electrical hysteresis loops for all the samples as a function of temperature. We found that the remanent ferroelectric polarization $P_{r}$ systematically increases with decreasing $r_{R}$, and thus $o$ - $\mathrm{LuMnO}_{3}\left(\mathrm{HoMnO}_{3}\right)$ produces the largest (smallest) $P_{r}$ value of $0.17(0.068) \mu \mathrm{C} / \mathrm{cm}^{2}$ at $6 \mathrm{~K}$. Based on the local structure analysis, we suggest that the orthorhombic manganites with $E$-type spin order have higher polarization contributions from the $t_{2 \mathrm{~g}}$ than the $e_{\mathrm{g}}$ orbitals.

\section{EXPERIMENTALS}

\section{A. Sample preparation}

We have synthesized $o-R \mathrm{MnO}_{3}(R=\mathrm{Er}, \mathrm{Tm}, \mathrm{Yb}$, and $\mathrm{Lu}$ ) specimens under a high pressure of $5 \mathrm{GPa}$ at $1423 \mathrm{~K}^{22}$ and confirmed that all of them had the orthorhombic (Pbnm) structure at $300 \mathrm{~K}$ without any impurities. Crystal structures were extracted by Rietveld refinement using the GSAS program. All the specimens investigated have a density of at least $95 \%$ of their theoretical value. We made thin platelike samples with thickness of approximately $0.3 \mathrm{~mm}$ and used silver epoxy (EPTEK H20E) to make electrodes. We employed a PPMS ${ }^{\mathrm{TM}}$ (Quantum Design) to control temperature environment for the

hysteresis loop or $J_{p}$ measurements. The electrical hysteresis loop measurements were performed for all the samples in their as-grown states after cooling the samples without an electric field bias. However, to check whether the remanent electric polarization is sensitive to the leakage current or not, we have also annealed $o-\mathrm{HoMnO}_{3}$, which is the most leaky specimen among the $o-R \mathrm{MnO}_{3}$ series $\left(1.4 \times 10^{10} \Omega \mathrm{cm}\right.$ at $\left.5 \mathrm{~K}\right)$, at $350^{\circ} \mathrm{C}$ for 24 hours in an $\mathrm{N}_{2}$ atmosphere. The $o-\mathrm{HoMnO}_{3}$ specimen annealed in the $\mathrm{N}_{2}$ atmosphere showed increased resistivity $\left(1.8 \times 10^{10} \Omega \mathrm{cm}\right)$ at $5 \mathrm{~K}$, without inducing much change in the lattice constants and the magnetic transition temperatures. Although the experimental results for $o-\mathrm{HoMnO}_{3}$ annealed in various gas conditions are already published in Ref. 21, we reproduce the electrical hysteresis loops of the $\mathrm{N}_{2}$-annealed sample and the remanent polarization data of the as-grown and $\mathrm{N}_{2}$-annealed samples in Fig. 3 to compare with those of other $o-R \mathrm{MnO}_{3}$.

\section{B. The PUND method}

For the PUND method we applied a series of positive $(\mathrm{P} i, i=0-2)$ and negative $(\mathrm{N} i, i=0-2)$ electric pulses, as shown in Fig. 2(a). The first two pulses, P0 and N0, are used to fully align the ferroelectric domains. During the next two pulses, P1 and P2 (N1 and N2), two curves representing effective polarization changes are recorded in the Sawyer-Tower circuit, and they are subtracted to obtain the pure hysteretic parts of the hysteresis loop without being obscured by resistive or capacitive components. ${ }^{21}$ Moreover, by employing a short pulse, the maximum peak field for electric poling can be increased to better align the ferroelectric domains without inducing electrical break-down effects. In particular, the space charge effect is minimized as the sample is poled in isothermal conditions. These features enable
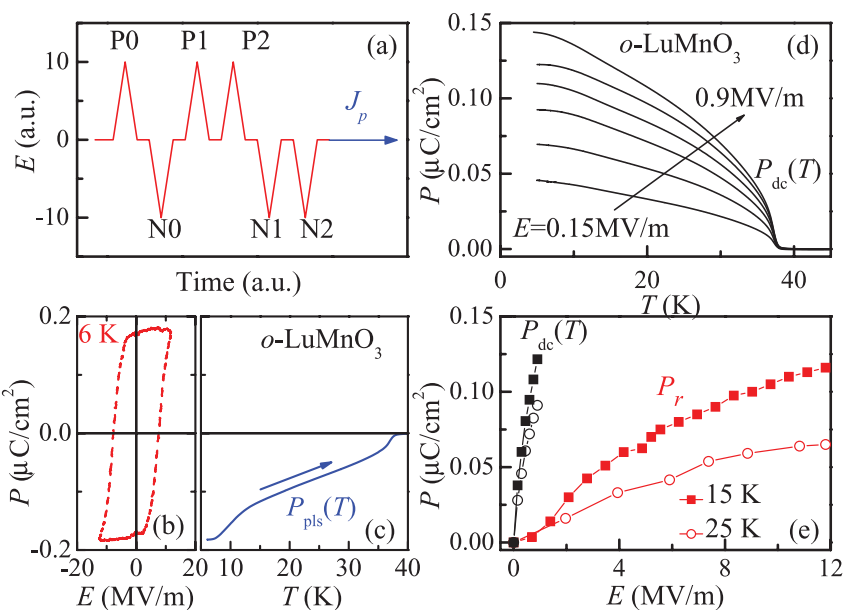

FIG. 2. (Color online) (a) Electrical pulse patterns used in the PUND method. (b) Typical hysteresis loop at $6 \mathrm{~K}$ and (c) $P_{\mathrm{pls}}(T)$ after the N2 pulse. (d) Temperature-dependent polarization $P_{\mathrm{dc}}(T)$ obtained by the conventional pyroelectric current measurements after dc electric field poling at $E=0.15,0.30,0.45,0.60,0.75$, and $0.9 \mathrm{MV} / \mathrm{m}$. (e) Remanent polarization $P_{r}$ vs peak electric field applied at 15 and $25 \mathrm{~K}$ for $o-\mathrm{LuMnO}_{3} . P_{\mathrm{dc}}(T)$ values at 15 and $25 \mathrm{~K}$ are also plotted as a function of applied dc electric field. 
us to mitigate the experimental problems encountered in conventional $J_{p}$ measurements with dc field poling. Based on the accurate capacitance and loss measurement with the capacitance bridge (Andeen Hagerling 2550A), we could also estimate the resistivity value of each sample as about $2 \times 10^{10}$ $\Omega \mathrm{cm}$ at $5 \mathrm{~K}$, larger than that of as-grown $o-\mathrm{HoMnO}_{3}$.

Figure 2(b) displays a typical electrical hysteresis loop obtained by this procedure for the case of $o-\mathrm{LuMnO}_{3}$ at $6 \mathrm{~K}$. The $y$-axis offset directly represents $P_{r} \approx 0.17 \mu \mathrm{C} / \mathrm{cm}^{2}$. To verify the thus obtained $P_{r}$ value by the PUND method, the pyroelectric current $J_{p}$ has been also measured after the short N2 pulse [Fig. 2(a)], and the temperature dependence of $P$, termed as $P_{\mathrm{pls}}(T)$ in Fig. 2(c), has been estimated. The difference between $P_{r}$ and $P_{\mathrm{pls}}(T)$ turns out to be less than $5 \%$ at $6 \mathrm{~K}$, supporting the conclusion that both $P_{r}$ and $P_{\mathrm{pls}}(T)$ are close to the intrinsic ferroelectric polarization. To estimate the intrinsic $P_{r}$ at each temperature, we have measured the hysteresis loop by increasing the amplitude of the pulse and then determined the polarization until the electrical breakdown happens. The $P_{r}$ vs maximum $E$ curves thus obtained for $o$ - $\mathrm{LuMnO}_{3}$ at $15 \mathrm{~K}$ and $25 \mathrm{~K}$ show almost saturation at the highest applied $E$ field of $11.8 \mathrm{MV} / \mathrm{m}$, suggesting that the saturated $P_{r}$ is close to the intrinsic polarization value measurable in the polycrystalline sample. On the other hand, the $P_{\mathrm{dc}}(T)$ curves determined through the $J_{p}$ measurement showed continual increase with the applied dc electric field poling without showing saturation [Fig. 2(d)], indicating that $P_{\mathrm{dc}}(T)$ contains large contributions from the space charges. As the result, the maximum polarization values obtained from $P_{\mathrm{dc}}(T)$ curves before an electrical breakdown at both $15 \mathrm{~K}$ and $25 \mathrm{~K}$ were clearly larger than those obtained using the PUND method [Fig. 2(e)]. Therefore, the conventional $P_{\mathrm{dc}}(T)$ measurements are likely to include significant contributions from trapped space charges that have accumulated during the dc poling process, while the $P_{\mathrm{pls}}(T)$ measurements do not.

\section{RESULTS}

By applying the PUND method, we first determined the hysteresis loops of two kinds of $o-\mathrm{HoMnO}_{3}$ samples, as-grown and $\mathrm{N}_{2}$-annealed ones, as summarized in Fig. 3. The as-grown $o-\mathrm{HoMnO}_{3}$ sample turned out to be less insulating than the annealed one so that the maximum allowed electric fields were 5 and $7 \mathrm{MV} / \mathrm{m}$ for the as-grown and annealed specimens, respectively. Related to this, the hysteresis loops of the asgrown sample [Fig. 3(a)] exhibited somewhat distorted shape and decreasing behavior with increase of electric field strength, making it difficult to judge whether the hysteresis loops are saturated or not. The apparently nonsaturating behavior seems to be associated with the enhanced leakage at high electric field regions in the as-grown sample. On the other hand, in the annealed sample [Fig. 3(b)] we could observe that all the hysteresis data more or less show saturating behavior at high electric field regions. As a result, the maximum applied electric field of $7 \mathrm{MV} / \mathrm{m}$ turned out to be much larger than the coercive electric fields of the annealed specimen in most of the measured temperatures to produce clearly saturated hysteresis loops. For example, the coercive electric field of the annealed sample at $15 \mathrm{~K}$ was found to be about $2.2 \mathrm{MV} / \mathrm{m}$, allowing almost full saturation at $7 \mathrm{MV} / \mathrm{m}$. Moreover, when

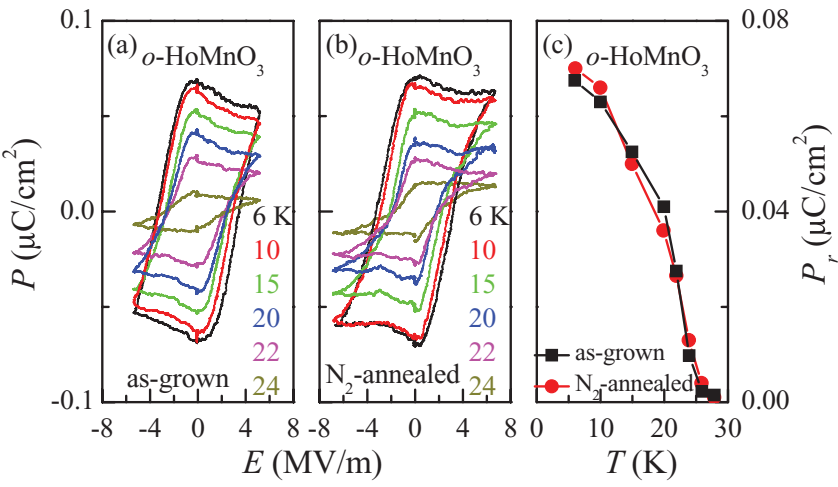

FIG. 3. (Color online) The electrical hysteresis loops at various temperatures for (a) the as-grown $o-\mathrm{HoMnO}_{3}$ and (b) the one annealed in the $\mathrm{N}_{2}$ atmosphere (reproduced from Ref. 21). (c) The resultant $P_{r}$ vs temperatures, showing no appreciable change (also reproduced from Ref. 21).

the $P_{r}$ vs temperature data were plotted for both as-grown and annealed samples in Fig. 3(c), we found that their $P_{r}$ values are almost the same, irrespective of the difference in their resistivity or the shape of the hysteresis loops. In Ref. 21 we also observed that the $P_{r}$ values were almost the same even after annealing in the $\mathrm{O}_{2}$ atmosphere. Therefore, we conclude that the $P_{r}$ values as obtained from the hysteresis loops measured by the PUND method are not sensitive to the leakage level of the specimen, thus indicating that the PUND method provides the intrinsic electric polarization of the specimen.

By applying the similar experimental method, we also determined the electrical hysteresis loops at various temperatures for other as-grown $o-R \mathrm{MnO}_{3}(R=\mathrm{Tm}, \mathrm{Yb}$, and $\mathrm{Lu})$ [Figs. 4(a)-4(c)] and summarized the resultant temperature dependence of $P_{r}$ and the $P_{\mathrm{pls}}(T)$ curves [Figs. 4(d)-4(f)]. Summarizing the hysteresis loops, appearing in Figs. 4(a)-4(c) as well as in Fig. 3(a), all the as-grown $o-R \mathrm{MnO}_{3}$ compounds showed development of remanent ferroelectric polarization at low temperatures. The onset temperatures of ferroelectric polarization were found to be $26,35,37$, and $38 \mathrm{~K}$ for $R=\mathrm{Ho}$, $\mathrm{Tm}, \mathrm{Yb}$, and $\mathrm{Lu}$, respectively, and they are consistent with the reported lock-in transition temperature, $T_{\mathrm{L}}$, in each compound. ${ }^{17,21,22}$ It is noteworthy that all the hysteresis loops appearing in Figs. 3 and 4 show saturating behavior at high electric fields except in very low temperature regions below $\sim 10-15 \mathrm{~K}$. Related to this observation, the coercive electric fields at temperatures above $15 \mathrm{~K}$ seem clearly lower than the maximum applied electric field in all the compounds. Moreover, the temperature dependence of $P_{r}$ and the $P_{\mathrm{pls}}(T)$ showed consistent values at almost all the temperatures in all the $o-R \mathrm{MnO}_{3}(R=\mathrm{Ho}, \mathrm{Tm}, \mathrm{Yb}$, and $\mathrm{Lu})$ specimens. We also showed in Ref. 21 that the annealed $o-\mathrm{HoMnO}_{3}$ specimens showed the consistent temperature-dependent $P_{r}$ and the $P_{\mathrm{pls}}(T)$.

\section{DISCUSSIONS}

Comparing the resultant $P_{r}$ values in $o-R \mathrm{MnO}_{3}$, to our surprise we found that the $o-\mathrm{LuMnO}_{3}$ specimen showed the 

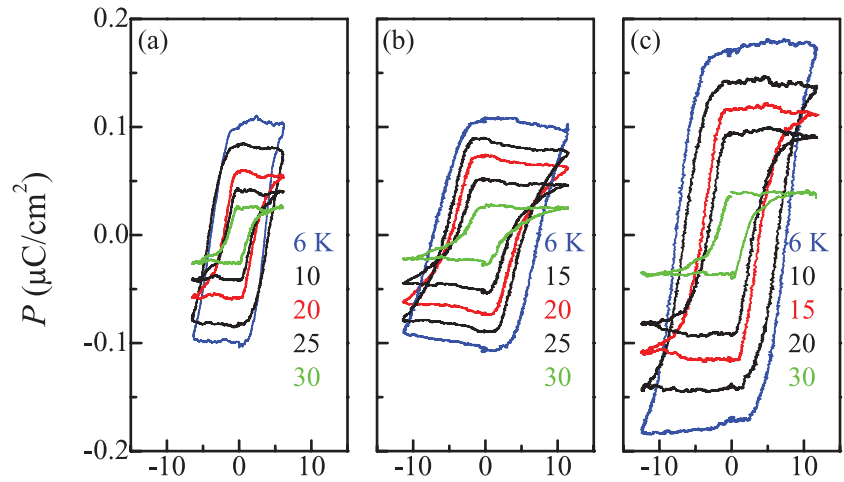

$E(\mathrm{MV} / \mathrm{m})$
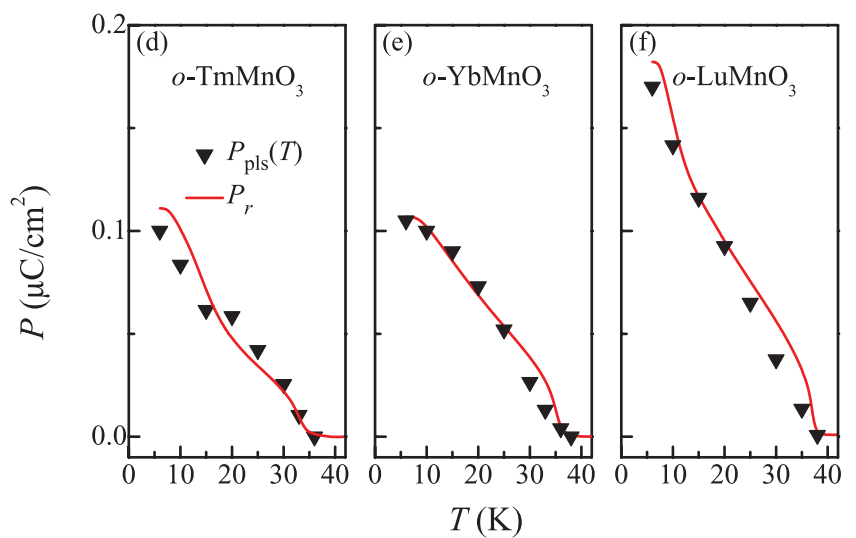

FIG. 4. (Color online) Electrical hysteresis loops of (a) $o$ $\mathrm{TmMnO}_{3}$, (b) $o-\mathrm{YbMnO}_{3}$, and (c) $o-\mathrm{LuMnO}_{3}$. Remanent polarization $P_{r}$ values and related $P_{\mathrm{pls}}(T)$ are summarized in (d)-(f).

largest $P_{r}$ value of $0.17 \mu \mathrm{C} / \mathrm{cm}^{2}$ at $6 \mathrm{~K}$. Upon extrapolation, $P_{r}$ would reach approximately $0.2 \mu \mathrm{C} / \mathrm{cm}^{2}$ at $0 \mathrm{~K}$ [Fig. 4(f)]. Because the $E$-type spin order is supposed to generate a uniaxial electric polarization along the $a$ axis, an ideal polycrystalline specimen, which is composed of crystalline grains with fully aligned ferroelectric domains inside, would have roughly one-third of the single crystal polarization value because of the random orientation of the grains. On the other hand, the procedure of an electric field poling can also generate less aligned ferroelectric domains inside the crystalline grain in practice because the poled electric field can be smaller than the effective coercive electric field required for each grain. The hysteresis loops at $6 \mathrm{~K}$, as shown in Figs. 3 and 4 , indeed do not seem to show clear saturation at such a low temperature. Therefore, $P_{r}=0.2 \mu \mathrm{C} / \mathrm{cm}^{2}$ at $6 \mathrm{~K}$ in our polycrystalline sample corresponds to a lower bound of $P_{a}=0.6 \mu \mathrm{C} / \mathrm{cm}^{2}$ in the $o-\mathrm{LuMnO}_{3}$ single crystal. We note that this $P_{a}$ value is still significantly larger than those observed in typical magnetic ferroelectrics, although it is still much lower than the theoretically predicted values of approximately $2-6 \mu \mathrm{C} / \mathrm{cm}^{2} .10,15$

In Figs. 4(d)-4(f) it is noted that $P_{r}$ values at low temperatures seem to decrease rather systematically as the rare earth radius is increased from $\mathrm{Lu}$ to Ho. To corroborate the observation, we also plotted the $P_{r}$ vs $T / T_{\mathrm{L}}$ for each compound in Fig. 5(a). In this plot with a scaled $x$ axis, the increase of $P_{r}$ with the decrease of $r_{R}$ becomes obvious below about $0.7 T_{\mathrm{L}}$.
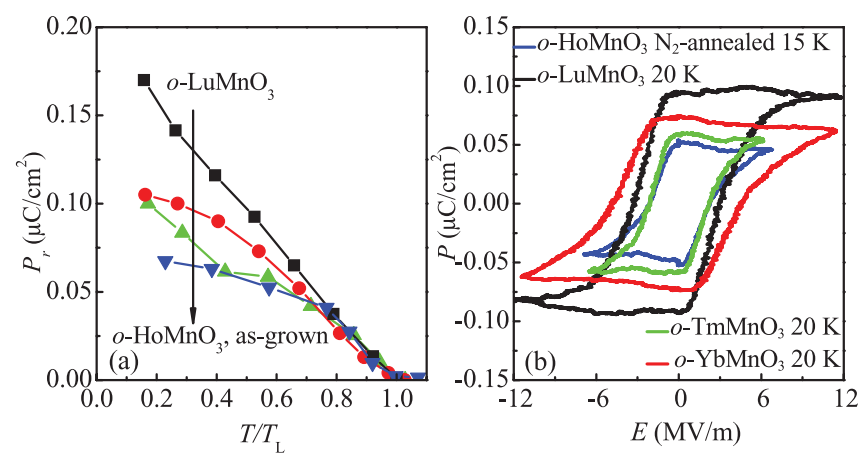

FIG. 5. (Color online) (a) The normalized $P_{r}$ vs $T / T_{\mathrm{L}}$ curves for the $o-\mathrm{HoMnO}_{3}, o-\mathrm{TmMnO}_{3}, o-\mathrm{YbMnO}_{3}$, and $o-\mathrm{LuMnO}_{3}$ samples (all as-grown states). (b) The electrical hysteresis loops at temperatures near $0.55 T_{\mathrm{L}}$ for the annealed $o-\mathrm{HoMnO}_{3}$ and the as-grown $o-\mathrm{TmMnO}_{3}, o-\mathrm{YbMnO}_{3}$, and $o-\mathrm{LuMnO}_{3}$ samples, which are replotted from the data presented in Figs. 3 and 4.

As the coercive electric fields of all the samples investigated were clearly much lower than the maximum applied electric fields above $\sim 15 \mathrm{~K}$, the systematic increase at such relatively high temperatures does not seem to come from the different amount of ferroelectric domain alignment in each compound. To clearly show this, we compare the hysteresis loops of all the samples at temperatures around $0.55 T_{\mathrm{L}}$ in Fig. 5(b), which demonstrates that each loop shows clearly saturating behavior. Moreover, even if the coercive electric fields at this temperature region are varying from $2-4 \mathrm{MV} / \mathrm{m}$ without showing any systematic change, the $P_{r}$ value systematically increases as we decrease $r_{R}$ from $\mathrm{HoMnO}_{3}$ to $\mathrm{LuMnO}_{3}$. It should be further noted in Fig. 5(b) that the $P_{r}$ value of $\sim 0.1 \mu \mathrm{C} / \mathrm{cm}^{2}$ at $20 \mathrm{~K}$ observed in $o-\mathrm{LuMnO}_{3}$ looks clearly larger than the possible variation of $P_{r}$ value in each compound from the somewhat distorted shape in its hysteresis loop. Therefore, we conclude that the observation of the systematic increase of $P_{r}$ value with decrease of $r_{R}$ is an intrinsic property of $o-R \mathrm{MnO}_{3}$ at least at relatively high temperatures above $T_{\mathrm{L}} / 2$.

Figure 6(a) summarizes the $3 P_{r}$ vs $r_{R}$ data obtained at temperatures of $6 \mathrm{~K}$ and $T_{\mathrm{L}} / 2$ in $o-R \mathrm{MnO}_{3}(R=\mathrm{Ho}-\mathrm{Lu})$. The $3 P_{r}$ values at $T_{\mathrm{L}} / 2$ were estimated by the interpolation from Fig. 5(a), demonstrating clearly that the $3 P_{r}$ values increase when the $r_{R}$ is reduced. Upon comparing those $3 P_{r}$ values of $o-\mathrm{HoMnO}_{3}$ and $o-\mathrm{LuMnO}_{3}$, we find that the $3 P_{r}$ values increase by 1.2 and 0.72 times at $6 \mathrm{~K}$ and $T_{\mathrm{L}} / 2$, respectively. Because the hysteresis loops around $T_{\mathrm{L}} / 2$ are clearly saturated at high electric fields, it is likely that the ferroelectric domains in each crystalline grain are close to the fully aligned states at $T_{\mathrm{L}} / 2$. Therefore, the smoothly increasing tendency of $3 P_{r}$ with $r_{R}$ at $T_{\mathrm{L}} / 2$ reflects the intrinsic $r_{R}$ dependence. It is also interesting to find that even at $6 \mathrm{~K}$, the increasing tendency of the $3 P_{r}$ values with $r_{R}$ are still maintained although the hysteresis loops are not fully saturated. Upon assuming that the degree of aligned ferroelectric domains is quite similar over all the measured $o-R \mathrm{MnO}_{3}$ at $6 \mathrm{~K}$, we postulate that the increasing tendency observed in the $3 P_{r}$ vs $r_{R}$ curve at $6 \mathrm{~K}$ still might reflect the intrinsic behavior of the system. The observation of the increasing $3 P_{r}$ with reduced $r_{R}$ is in contrast with the theoretical predictions in Ref. 10, which showed almost constant $P$ behavior regardless of the $r_{R}$ change. Furthermore, 


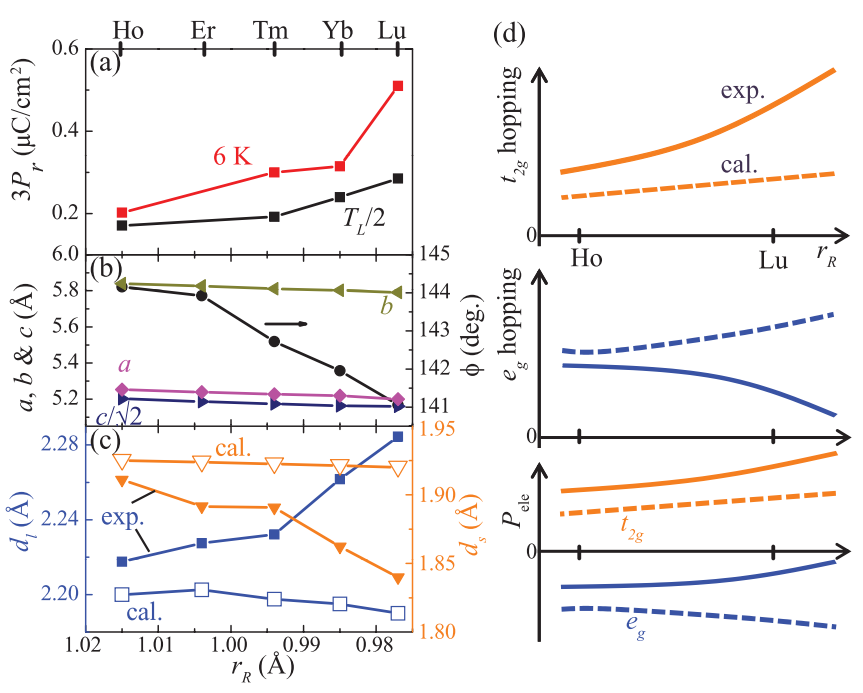

FIG. 6. (Color online) The rare earth ionic radius $r_{R}$ dependence of (a) $3 P_{r}$ values at $6 \mathrm{~K}$ and $T_{\mathrm{L}} / 2$, (b) lattice constants and bond angle $(\phi)$, and (c) experimental $d_{s}$ and $d_{l}$ values (solid symbols) at room temperature and calculated average $d_{s}$ and $d_{l}$ (open symbols) (from Ref. 10). (d) Schematic diagram for expected hopping integrals for $t_{2 g}$ (top panel) and $e_{g}$ orbitals (middle panel) and related electronic polarization contributions (bottom panel). Solid and dash lines represent the expected behaviors based on the experimental data in this work and previously calculated lattice parameters (Ref. 10), respectively.

our results are also inconsistent with the experimental data in Ref. 18 , in which the conventional $J_{p}$ measurements after the dc electric poling procedure resulted in $P$ values that were almost constant over different $r_{R}$.

In order to understand the origin of this change of $3 P_{r}$ with $r_{R}$ shown in Fig. 6(a), we determined the structural parameters of all the samples at $300 \mathrm{~K}$, and the results are summarized in Figs. 6(b) and 6(c). The resultant lattice constants and in-plane $\mathrm{Mn}-\mathrm{O}-\mathrm{Mn}$ bond angle $\phi$ are quite similar to the reported experimental values. ${ }^{22}$ Indeed, the $\phi$ value systematically decreases with $r_{R}$, showing that the compounds with a smaller $r_{R}$ result in a more distorted local structure. On the other hand, the Mn-O bond lengths, $d_{l}$ and $d_{s}$, clearly show opposite tendency with respect to the variation of $r_{R} ; d_{l}\left(d_{s}\right)$ increases (decreases) with the decrease in $r_{R}$ [Fig. 6(c)]. This experimental finding is consistent with other experiment results ${ }^{22}$ but is in contrast with the behavior of the input parameters used in the first principles calculation, ${ }^{10}$ in which the optimized crystal structure in the $E$-type spin order results in the decreasing behavior for both $d_{l}$ and $d_{s}$ with respect to the $r_{R}$ decrease. Figure 6(c) compares these contrasting experimental and theoretical behaviors of $d_{l}$ and $d_{s}$ with $r_{R}$. Moreover, it is noted that the experimental changes of $d_{l}$ and $d_{s}$ over $r_{R}$, which are approximately 3\%, are much bigger than the theoretical predictions, which are less than $0.5 \%$. These lattice parameter variations over $r_{R}$ are thus expected to hold even at low temperatures because thermal shrinking in these $o-R \mathrm{MnO}_{3}$ compounds is estimated to be less than $0.3 \%$ between 300 and $10 \mathrm{~K}^{17}$

The first principles calculation in Ref. 10 discussed how the input structural parameters can crucially affect the electronic polarization $P_{\text {ele }}$, which is dominant over the ionic polar- ization, $P_{\text {ion }}$. First, with decreasing $d_{l}$, the hopping integral between the $e_{g}$ orbitals increases and thus the $e_{g}$ contribution to $P_{\text {ele }}$ increases. Second, the decrease of $d_{s}$ can enhance the hopping between $t_{2 g}$ orbitals so that the $t_{2 g}$ contribution to $P_{\text {ele }}$ will increase too. Because the enhanced contributions of $e_{g}$ and $t_{2 g}$ orbitals are opposite in sign, the total $P$ should eventually become almost independent of $r_{R}$ and then become close to $6 \mu \mathrm{C} / \mathrm{cm}^{2}$ in all the $o-R \mathrm{MnO}_{3}(R=$ Ho to $\mathrm{Lu}){ }^{10}$ The dashed lines in Fig. 6(d) schematically describe these theoretical predictions for the hopping integrals and related contributions to $P_{\text {ele }}$.

On the other hand, our new experimental results for $d_{l}$ and $d_{s}$ in Fig. 6(c) suggest a new scenario that with decreasing $r_{R}$, the hopping integral between $e_{g}$ orbitals should be suppressed significantly while that between $t_{2 g}$ orbitals should be increased [solid lines in Fig. 6(d)]. Accordingly, we can expect that the $t_{2 g}$ orbitals contribute to $P_{\text {ele }}$ more significantly than $e_{g}$ orbitals overall, and this tendency will increase more as $R$ changes from Ho to Lu [Fig. 6(d)]. As the ionic and oxygen contributions are relatively small and nearly independent of $r_{R}$, the total $P$, which is dominated by $P_{\text {ele }}\left(t_{2 \mathrm{~g}}\right.$ orbital contribution), will be then enhanced as $r_{R}$ decreases. The observed increase of $3 P$ from $o-\mathrm{HoMnO}_{3}$ to $o-\mathrm{LuMnO}_{3}$ seems consistent with this qualitative explanation based on the existing theoretical prediction. On the other hand, Okuyama et $a l .{ }^{23}$ recently found in a single crystal of $o-\mathrm{YMnO}_{3}$ that the experimental $\mathrm{Mn}$ and $\mathrm{O}$ displacements along the $a$ axis below the $E$-type spin ordering temperature show opposite directions and at least four times smaller as compared with the theoretical predictions. ${ }^{10}$ This specific observation in $o-\mathrm{YMnO}_{3}$ then implies that even in other $o-R \mathrm{MnO}_{3}$ with $E$-type spin order, the net $P_{\text {ion }}$ contribution to the total $P$ can be even smaller than the theoretical prediction, and that the contribution of the $e_{\mathrm{g}}\left(t_{2 \mathrm{~g}}\right)$ orbital can be overestimated (underestimated) even to the $P_{\text {ion }}$. Therefore, it will be worth further theoretical investigation based on the structural and electrical information provided here to see whether the existing theoretical framework is still valid or requires other explanations to understand the observed polarization value and its rare earth dependence in $o-R \mathrm{MnO}_{3}$.

\section{CONCLUSION}

To conclude, we determined accurately the ferroelectric polarization in $o-R \mathrm{MnO}_{3}(R=\mathrm{Ho}, \mathrm{Tm}, \mathrm{Yb}$, and $\mathrm{Lu})$ with $E$-type spin order by using the PUND method. The obtained polarization values increase systematically upon reducing the rare earth ionic radius from $R=$ Ho to $\mathrm{Lu}$, and the maximum ferroelectric polarization value at $0 \mathrm{~K}$ is estimated to be at least $0.6 \mu \mathrm{C} / \mathrm{cm}^{2}$ in $o-\mathrm{LuMnO}_{3}$. Our structural analyses imply that $t_{2 g}$ rather than $e_{\mathrm{g}}$ orbitals play a more crucial role in determining ferroelectric polarization.

\section{ACKNOWLEDGMENTS}

We thank Y. Liu for helpful discussion. This work was financially supported by the National Creative Research Initiative (2010-0018300) and the Fundamental R\&D Program for Core Technology of Materials of MOKE. The work at IOP CAS was supported by NSF \& MOST of China through research projects. 
*optopia@snu.ac.kr

${ }^{1}$ T. Kimura, N. Goto, H. Shintani, T. Arima, and Y. Tokura, Nature (London) 426, 55 (2003).

${ }^{2}$ N. Hur, S. Park, P. A. Sharma, J. S. Ahn, S. Guha, and S.-W. Cheong, Nature (London) 429, 392 (2004).

${ }^{3}$ M. Fiebig, J. Phys. D 38, R123 (2005).

${ }^{4}$ D. I. Khomskii, J. Magn. Magn. Mater. 306, 1 (2006).

${ }^{5}$ S.-W. Cheong and M. Mostovoy, Nat. Mater. 6, 13 (2007).

${ }^{6}$ L. C. Chapon, G. R. Blake, M. J. Gutmann, S. Park, N. Hur, P. G. Radaelli, and S.-W. Cheong, Phys. Rev. Lett. 93, 177402 (2004).

${ }^{7}$ Y. J. Choi, H. T. Yi, S. Lee, Q. Huang, V. Kiryukhin, and S.-W. Cheong, Phys. Rev. Lett. 100, 047601 (2006).

${ }^{8}$ M. Kenzelmann, A. B. Harris, S. Jonas, C. Broholm, J. Schefer, S. B. Kim, C. L. Zhang, S.-W. Cheong, O. P. Vajk, and J. W. Lynn, Phys. Rev. Lett. 95, 087206 (2005).

${ }^{9}$ T. Arima, A. Tokunaga, T. Goto, H. Kimura, Y. Noda, and Y. Tokura, Phys. Rev. Lett. 96, 097202 (2006).

${ }^{10}$ K. Yamauchi, F. Freimuth, S. Blugel, and S. Picozzi, Phys. Rev. B 78, 014403 (2008).

${ }^{11}$ K. Yamauchi and S. Picozzi, J. Phys. Condens. Mat. 21, 064203 (2009).

${ }^{12}$ I. A. Sergienko, C. Sen, and E. Dagotto, Phys. Rev. Lett. 97, 227204 (2006).

${ }^{13}$ S. Picozzi, K. Yamauchi, B. Sanyal, I. A. Sergienko, and E. Dagotto, Phys. Rev. Lett. 99, 227201 (2007).
${ }^{14}$ Y. Murakami, J. P. Hill, D. Gibbs, M. Blume, I. Koyama, M. Tanaka, H. Kawata, T. Arima, Y. Tokura, K. Hirota, and Y. Endoh, Phys. Rev. Lett. 81, 582 (1998).

${ }^{15}$ A. Stroppa and S. Picozzi, Phys. Chem. Chem. Phys. 12, 5405 (2010).

${ }^{16}$ B. Lorenz, Y. Q. Wang, and C. W. Chu, Phys. Rev. B 76, 104405 (2007).

${ }^{17}$ V. Yu. Pomjakushin, M. Kenzelmann, A. Dönni, A. B. Harris, T. Nakajima, S. Mitsuda, M. Tachibana, L. Keller, J. Mesot, H. Kitazawa, and E. T. Muromachi, New J. Phys. 11, 043019 (2009).

${ }^{18}$ S. Ishiwata, Y. Kaneko, Y. Tokunaga, Y. Taguchi, T. Arima, and Y. Tokura, Phys. Rev. B 81, 100411(R) (2010).

${ }^{19}$ J. F. Scott, L. Kammerdiner, M. Parris, S. Traynor, V. Ottenbacher, A. Shawabkeh, and W. F. Oliver, J. Appl. Phys. 64, 787 (1988).

${ }^{20}$ M. Fukunaga and Y. Noda, J. Phys. Soc. Jpn. 77, 064706 (2008).

${ }^{21}$ S. M. Feng, Y. S. Chai, J. L. Zhu, N. Manivannan, Y. S. Oh, L. J. Wang, Y. S. Yang, C. Q. Jin, and K. H. Kim, New J. Phys. 12, 073006 (2010).

${ }^{22}$ M. Tachibana, T. Shimoyama, H. Kawaji, T. Atake, and E. T. Muromachi, Phys. Rev. B 75, 144425 (2007).

${ }^{23}$ D. Okuyama, S. Ishiwata, Y. Takahashi, K. Yamauchi, S. Picozzi, K. Sugimoto, H. Sakai, M. Takata, R. Shimano, Y. Taguchi, T. Arima, and Y. Tokura, Phys. Rev. B 84, 054440 (2011). 